\title{
Can Prolactinemia Cause Coronary Thrombosis
}

\author{
Goutham Akidi ${ }^{1}$ \\ ${ }^{1}$ Department of Cardiology, Nizam's Institute of Medical Sciences, \\ Punjagutta, Hyderabad, Telangana, India
}

\begin{abstract}
Address for correspondence Goutham Akidi, MD, Department of Cardiology, Nizam's Institute of Medical Sciences, Punjagutta, Hyderabad, Telangana 500082, India (e-mail: gouthamreddyakidi@gmail.com).
\end{abstract}

Ind J Car Dis Wom 2020;5:48-52

\begin{abstract}
Keywords

- coronary thrombosis

- prolactinemia

There is an increasing trend of myocardial infarction (MI) among the young population. There exist various causes for this trend, as the pathogenesis of increased $\mathrm{MI}$ could be atheromatous, nonatheromatous, or hypercoagulable, and involve substance misuse. Here, we report a case of a 32-year-old female patient presented with coronary thrombosis; on evaluation, she was found to have hyperprolactinemia with no other causes of coronary thrombosis. Hyperprolactinemia is a rare cause of recurrent coronary thrombosis. Prolactin levels are even elevated in MI but they return to normal values in 2 to 4 weeks; however, this patient experienced a persistent elevation of prolactin levels with no signs of pituitary adenoma, so there is a possibility that elevated prolactin levels could cause coronary thrombosis.
\end{abstract}

\section{Introduction}

Young myocardial infarction (MI) is increasingly trending in the present era when a coronary artery disease (CAD) epidemic seems to have broken out in India. The disease in such a young age will not only exert financial and psychological effects but also lead to significant morbidity. The pathophysiology behind this acute coronary syndrome (ACS) is different among young people.

In patients who are less than 45 years of age, causes of MI are divided into four groups:

(1) CAD with atheromatosis.

(2) CAD with nonatheromatous changes.

(3) Hypercoagulable states.

(4) Substance misuse-related myocardial infarction.

There is a significant overlap in the pathogenesis of different age groups.

Reporting a case of prolactinemia with myocardial infarction and acute presentation.

\section{Case Report}

Reporting a case of young female with acute MI: a 32-year-old female patient with no risk factors presented to our hospital with the complaints of chest pain of 8 hours duration. The chest pain was typical retrosternal, associated with the diaphoresis squeezing type and radiating to upper left limb and dyspnea of NYHA class II. The patient was admitted to the cardiac ICCU where she was treated for ACS.

The patient was hemodynamically stable and ECG suggested sinus rhythm with T-wave inversions in V1 to V6 and lead 1, 2 and 3, aVL and aVF, ST depression in v1 to v6.

ECHO suggested global LV hypokinesia, moderate LV dysfunction, and mild mitral regurgitation.

The patient was taken to the Cath laboratory and coronary angiogram suggested thrombotic occlusion of left circumflex proximal portion (LCX), obtuse marginal (OM) branch, and midportion of the left anterior descending artery (LAD) ( - Figs. 1 and 2 ). Stenting was deferred because of multiple sites of thrombus in different coronary vascular territories (LCX and LAD), and the patient was treated with an infusion of glycoprotein IIB IIIA inhibitors (ABCIXIMAB), dual antiplatelets, $\beta$-blockers, and low-dose diuretics for 12 hours. Later, the patient was reviewed with the coronary angiogram which showed the cleared thrombus in the LCX and OM and distal thrombus in the LAD with TIMI 2 flow ( - Figs. 3 and 4 ). The patient was evaluated for hypercoagulable state and other causes of simultaneous multiple thrombo-embolism of coronary vessels. The patient remained asymptomatic with medical management.

The patient does not give a history of oral contraceptives intake. Repeat transthoracic and transesophageal echo did not reveal any cardiac source of embolism or presence of
(C)2020 Women in Cardiology and Related Sciences
License terms

() (1) $\Theta \circledast$ 


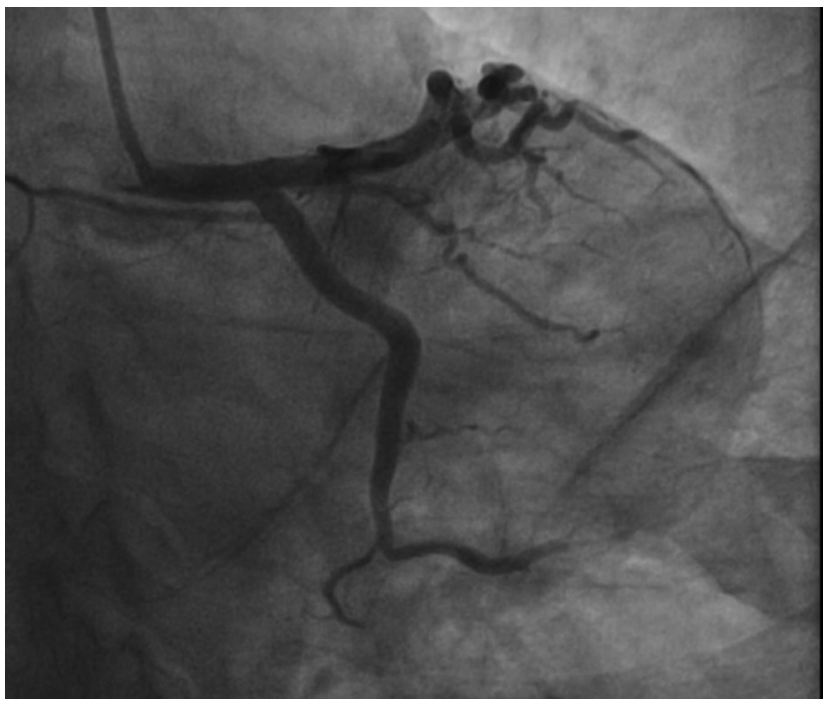

Fig. 1 LAO caudal view showing thrombus in the LCX and OM. Abbreviations: OM, obtuse marginal artery; LAO, left anterior oblique; LCX, left circumflex artery.

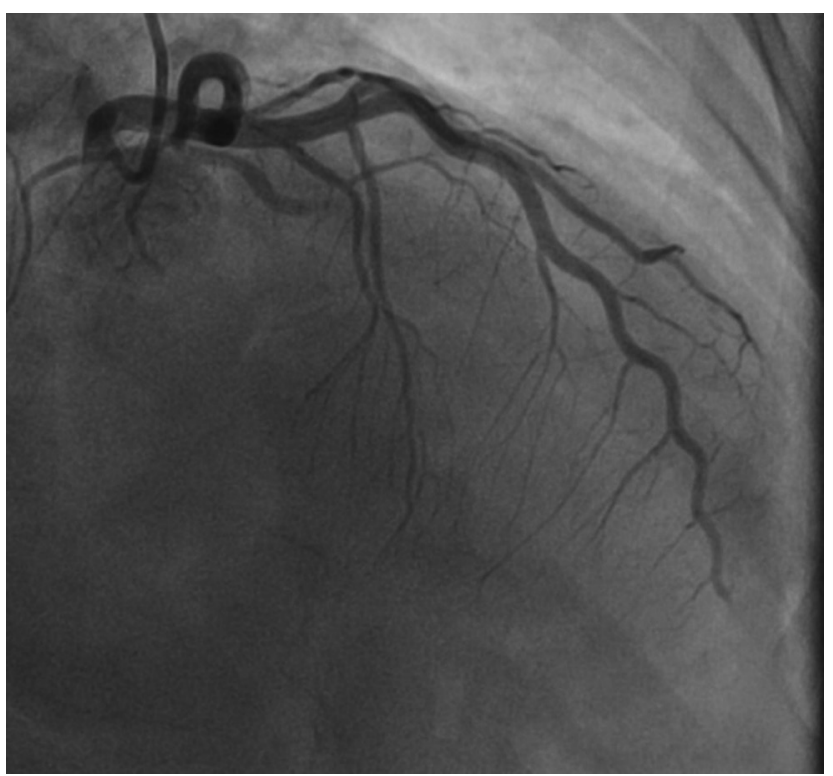

Fig. 2 RAO cranial image showing mid to distal LAD thrombus. Abbreviations: LAD, left anterior descending; RAO, right anterior oblique.

patent foramen oval. On evaluation, the patient was found out to have normal homocysteine, antithrombin, protein C, protein $\mathrm{S}$ levels, negative for Factor V Leiden and prothrombin mutation, and normal thyroid levels. The patient was clinically anemic with hemoglobin of $7.8 \mathrm{~g} / \mathrm{dl}$, and iron profile showing iron-deficiency anemia picture. Serum prolactin was $90.34 \mathrm{ng} / \mathrm{ml}$ and repeated prolactin levels after 6 weeks were $67.65 \mathrm{ng} / \mathrm{mL}$, both values were higher than normal for her age and condition, without clinical signs prolactinomas. The patient was advised MRI of the brain to look for pituitary tumors but the patient did not undergo the procedure, and in this case, either elevation of prolactin could be a cause of the multiple coronary thromboses, and microadenoma of the pituitary maybe a cause of raised prolactinomas.

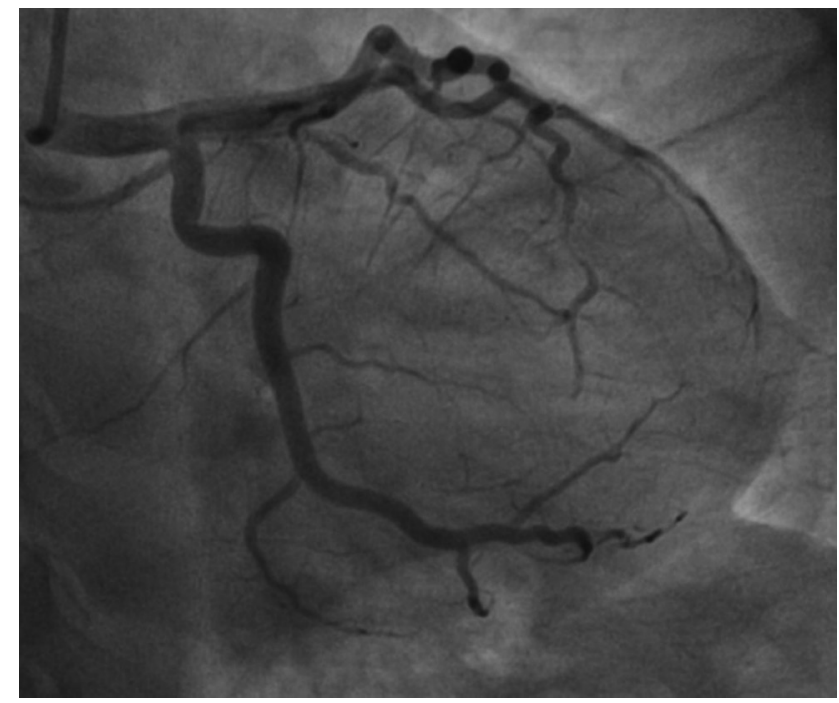

Fig. 3 Repeat angiogram of the same patient showing cleared thrombus in LCX and OM after GP 2b 3a infusion. Abbreviation: Abbreviations: $\mathrm{OM}$, obtuse marginal artery; LCX, left circumflex artery.

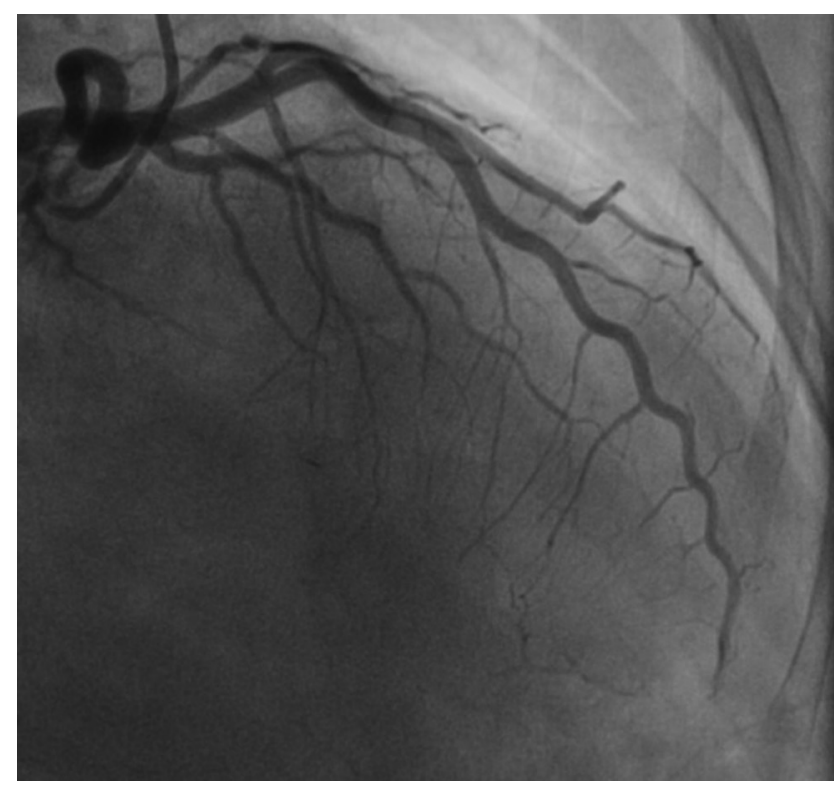

Fig. 4 Repeat angiogram showing still showing there is a mild distal LAD thrombus. Abbreviation: LAD, left anterior descending.

\section{Discussion}

In the world, the incidence of coronary heart disease (CHD) is declining in all age groups. The prevalence of the disease was found to be around $20 \%$ in men and women, and in the age group of 30 to 45 years, the incidence is less than $1 \% .{ }^{1}$ Actual figures in young patients may be lesser, due to atypical presentation and they are not willing to submit themselves for further evaluation. ${ }^{2}$ It was found that CHD in population below the age of 40 years represents only $3 \%$ of all patients with CHD. ${ }^{3}$

In the young population, MI can be divided into two groups:

(1) Those with normal coronary arteries on angiogram.

(2) Those with coronary artery disease of varying etiology. 
There is a significant overlap in pathophysiology between these two different groups.

\section{Acute Myocardial Infarction In Angiographically "Normal” Coronary Arteries}

In the presence of normal coronaries, pathophysiology of MI is unclear, but can be explained with different etiologies like coronary artery thrombosis, spasm, embolization, or sometimes a combination of these. Hypercoagulable states are like antiphospholipid syndrome, protein S and protein C, Factor XII deficiency, homocystinemia, and nephrotic syndrome. ${ }^{4-7}$ Coronary embolization is very rare but is seen with the aortic valve-infective endocarditis. ${ }^{8}$ The spasm of a coronary artery is seen with substance misuse like the recreational use of cocaine, ${ }^{9,10}$ amphetamine use, and alcohol binge drinking. ${ }^{11-13}$

\section{Hypercoagulable States \\ Nephrotic Syndrome}

In nephrotic syndrome, there is loss of proteins in the urine, and loss of anticoagulant proteins will lead to increase in the rates of thrombosis. There is a loss of factors like factor IX, XI, XII which are decreased due to urinary loss. ${ }^{11}$ In this scenario, the liver tries to compensate with increased synthesis of different proteins like II, VII, VIII, X, XIII, and fibrinogen resulting in raised blood levels. ${ }^{14-16}$ There is a significant reduction in antithrombin III, protein S and protein C, which leads to increased thrombosis of vessels.

\section{Antiphospholipid Syndrome (Hughes' Syndrome)}

Antiphospholipid syndrome involves associating antiphospholipid antibodies with both arterial and venous thrombosis together with loss of pregnancy in the second trimester. ${ }^{17}$ The antibodies associated with the syndrome are the lupus anticoagulant and anticardiolipin antibody, and IgG antibodies against plasma phospholipid-binding proteins such as alfa 2-glycoprotein I and prothrombin are the main antiphospholipid antibodies involved in thrombosis and atherosclerosis of blood vessels. Cardiac complications included in this syndrome are Mis and valvular abnormalities of varying degrees. ${ }^{18-20}$ However, there is evidence that premature atherosclerosis in the syndrome increases evidence of platelet adhesiveness that is mainly due to anticardiolipin antibodies and which, in turn, leads to an increase in the possibility of MI along with increased thrombosis.

\section{Coronary Artery Spasm}

Cocaine-The cardiac effects of cocaine are mediated via four main pathways ${ }^{21,22}$ :

(1) Increased heart rate and blood pressure leads to increased oxygen demand which, in turn, leads to MI.

(2) Cocaine has an $\alpha 1$ adrenergic property which leads to coronary vasoconstriction and one more mechanism of calcium-dependent vasoconstriction.

(3) Cocaine leads to endothelial dysfunction which, in turn, leads to vasoconstriction and thrombosis.

(4) Promotion of arteriosclerosis. ${ }^{23}$
A predominant mechanism for MI in Cocaine has coronary vasospasm which leads to simple angina and infarction of myocardium. With the use of cocaine, there are reports of arrhythmias like tachyarrhythmias and bradyarrhythmia and even sudden cardiac death; myocardial contraction bands are the probable cause for such effects. ${ }^{24}$

\section{Myocardial Bridging}

Myocardial bridging is a condition in which part of a coronary vessel runs under the subepicardial myocardium or a band of myocardium overlying the epicardial coronary vessel, which is a congenital anomaly. This can lead to decreased blood flow during systole that can even persist during diastole, resulting in ischemia of myocardium, which leads to MI. Treatment of bridging involves surgical splitting of the band and recent reports suggest even implantation of a stent. ${ }^{25}$

\section{Myocardial Infarction Associated With The Angiographically "Abnormal" Coronary Arteries}

Even the normal coronary arteries angiographically may have high-burden of plaque when assessed using intracoronary ultrasound. The fractions of MI include atherosclerosis, arterial aneurysms, arterial ectasia, spontaneous dissections of coronary vessels, and anomalous origin of coronary arteries.

\section{Accelerated Atherosclerosis}

Among young individuals who are less than 45 years of age, accelerated atherosclerosis can be causative factor for MI. Various factors like familial hypercholesterolemia, smoking, obesity, inactive habitus, family history can be the causative factors. ${ }^{26-30}$

\section{Spontaneous Dissections}

In the young age group, it can be one of the rare causes of MI, which is common among young females. The most common artery involved is the LAD artery. Dissection plane in spontaneous dissection is in between adventia and media, and treatment option is either surgery or stenting the dissected part. ${ }^{31}$

\section{Coronary Aneurysms, Coronary Ectasia, and Anomalous Coronary Vessel Origin}

These conditions can be a rare cause of the infarction in young people; however, the exact mechanism of how it causes infarction of myocardium in young is not clear. ${ }^{32,33}$

\section{Hyperprolactinemia}

Hyperprolactinemia may due to acute-phase reaction to coronary syndrome. It is a neuroendocrine stress reaction to ACSs, or hyperprolactinemia, which may lead to the development of ACS through various mechanisms that induce acute endothelial dysfunction, insulin resistance, and induction of vascular immune reactions. Therefore, prolonged hyperprolactinemia leads to arteriosclerosis, augmentation of arterial stiffness, and hypertension. ${ }^{34}$ 
Studies have shown that prolactin augments adhesion of the immunecells into endothelium through integrin-mediated effects that cause proliferation of smooth muscle cells of the vessel which may contribute to atherosclerotic expansion and elevation of cardiac risk profile. ${ }^{35}$ Physiological prolactin levels stimulate JAK-STAT pathway which, in turn, stimulate cardiomyocyte hypertrophy, angiogenesis, expression of prolactin receptors, and cardiac protection via upregulation of superoxide dismutase that inhibit free radical formations, but excessive prolactin above physiological level leads to severe inhibition of cardiac metabolism and damaging of cardiac microvasculatures which may inhibit, via dopamine agonist, prolactin secretions. ${ }^{36}$

All these observations showed that a high-prolactin level may lead to the development of an ischemic heart disease, also, hyperprolactinemia leads to dyslipidemia, augmentation of platelets aggregation, and amplification of vascular thrombosis that leads to an increase in the risk score of an ACS. ${ }^{37}$

Besides, high-prolactin serum level causes significant vasoconstriction and induction of oxidative stress in the coronary vessels, since prolactin receptors are overexpressed in atherosclerotic plaque macrophage which indicates the association between prolactin and induction of inflammatory markers that may explain the connection between serum prolactin and cardiovascular mortality.

\section{Conclusion}

Even though in this case the direct association of hyperprolactinemia and ACS cannot be proved, with the evidence of previous literature and absence of other predisposing factors, it lends support to the theory that this young female had multiple site coronary thromboses due to hyperprolactinemia.

\section{Conflict of Interest}

None.

\section{References}

1 British Heart Foundation. Coronary Heart Disease Statistics 2012. Available at: https://www.bhf.org.uk/informationsupport/ publications/statistics/coronary-heart-disease-statistics-2012. Accessed March 12, 2020

2 Klein LW, Nathan S. Coronary artery disease in young adults. J Am Coll Cardiol 2003;41(4):529-531

3 Jalowiec DA, Hill JA. Myocardial infarction in the young and in women. Cardiovasc Clin 1989;20(1):197-206

4 Fujimura O, Gulamhusein S. Acute myocardial infarction: thrombotic complications of nephrotic syndrome. Can J Cardiol 1987;3(6):267-269

5 Hamsten A, Norberg R, Björkholm M, de Faire U, Holm G. Antibodies to cardiolipin in young survivors of myocardial infarction: an association with recurrent cardiovascular events. Lancet 1986;1(8473):113-116

6 Manzar KJ, Padder FA, Conrad AR, Freeman I, Jonas EA. Acute myocardial infarction with normal coronary artery: a case report and review of literature. Am J Med Sci 1997;314(5):342-345

7 Penny WJ, Colvin BT, Brooks N. Myocardial infarction with normal coronary arteries and factor XII deficiency. Br Heart J 1985;53(2):230-234
8 Agirbasli MA, Hansen DE, Byrd BF III. Resolution of vegetations with anticoagulation after myocardial infarction in primary antiphospholipid syndrome. J Am Soc Echocardiogr 1997;10(8):877-880

9 Simpson RW, Edwards WD. Pathogenesis of cocaine-induced ischemic heart disease. Autopsy findings in a 21-year-old man. Arch Pathol Lab Med 1986;110(6):479-484

10 Ross GS, Bell J. Myocardial infarction associated with inappropriate use of topical cocaine as treatment for epistaxis. Am J Emerg Med 1992;10(3):219-222

11 Williams MJA, Restieaux NJ, Low CJS. Myocardial infarction in young people with normal coronary arteries. Heart 1998;79(2):191-194

12 Moreyra AE, Kostis JB, Passannante AJ, Kuo PT. Acute myocardial infarction in patients with normal coronary arteries after acute ethanol intoxication. Clin Cardiol 1982;5(7):425-430

13 Huang $\mathrm{CN}, \mathrm{Wu} \mathrm{DJ}$, Chen KS. Acute myocardial infarction caused by transnasal inhalation of amphetamine. Jpn Heart J 1993;34(6):815-818

14 Fahal IH, McClelland P, Hay CR, Bell GM. Arterial thrombosis in the nephrotic syndrome. Postgrad Med J 1994;70(830):905-909

15 Kendall AG, Lohmann RC, Dossetor JB. Nephrotic syndrome. A hypercoagulable state. Arch Intern Med 1971;127(6):1021-1027

16 Takeda Y, Chen AY. Fibrinogen metabolism and distribution in patients with the nephrotic syndrome. J Lab Clin Med 1967;70(4):678-685

17 Hughes GR. Thrombosis, abortion, cerebral disease, and the lupus anticoagulant. Br Med J(Clin Res Ed) 1983;287(6399):1088-1089

18 Jouhikainen T, Pohjola-Sintonen S, Stephansson E. Lupus anticoagulant and cardiac manifestations in systemic lupus erythematosus. Lupus 1994;3(3):167-172

19 Khamashta MA, Cervera R, Asherson RA, et al. Association of antibodies against phospholipids with heart valve disease in systemic lupus erythematosus. Lancet 1990;335(8705):1541-1544

20 Espínola-Zavaleta N, Vargas-Barrón J, Colmenares-Galvis T, et al. Echocardiographic evaluation of patients with primary antiphospholipid syndrome. Am Heart J 1999;137(5):973-978

21 Pitts WR, Lange RA, Cigarroa JE, Hillis LD. Cocaine-induced myocardial ischemia and infarction: pathophysiology, recognition, and management. Prog Cardiovasc Dis 1997;40(1):65-76

22 Flores ED, Lange RA, Cigarroa RG, Hillis LD. Effect of cocaine on coronary artery dimensions in atherosclerotic coronary artery disease: enhanced vasoconstriction at sites of significant stenoses. J Am Coll Cardiol 1990;16(1):74-79

23 Kolodgie FD, Virmani R, Rice HE, et al. Intravenous cocaine accelerates atherosclerosis in cholesterol-fed New Zealand white rabbits, abstracted. J Am Coll Cardiol 1990;15(suppl A): $217 \mathrm{~A}$

24 Castro VJ, Nacht R. Cocaine-induced bradyarrhythmia: an unsuspected cause of syncope. Chest 2000;117(1):275-277

25 Klues HG, Schwarz ER, vom Dahl J, et al. Disturbed intracoronary hemodynamics in myocardial bridging: early normalization by intracoronary stent placement. Circulation 1997;96(9):2905-2913

26 McGill HC Jr, McMahan CA, Zieske AW, et al. Association of coronary heart disease risk factors with microscopic qualities of coronary atherosclerosis in youth. Circulation 2000;102(4):374-379

27 Vuorio AF, Kontula K, Turtola H, Sajantila A. Post mortem molecularly defined familial hypercholesterolemia and sudden cardiac death of young men. Forensic Sci Int 1999;106(2):87-92

28 Booth GL, Wang EE. Preventive health care, 2000 update: screening and management of hyperhomocysteinemia for the prevention of coronary artery disease events. The Canadian Task Force on Preventive Health Care. CMAJ 2000;163(1):21-29

29 Horejsí B, Ceska R. Apolipoproteins and atherosclerosis. Apolipoprotein E and apolipoprotein(a) as candidate genes 
of premature development of atherosclerosis. Physiol Res 2000;49(Suppl 1) :S63-S69

30 Gaeta G, De Michele M, Cuomo S, et al. Arterial abnormalities in the offspring of patients with premature myocardial infarction. N Engl J Med 2000;343(12):840-846

31 Vale PR, Baron DW. Coronary artery stenting for spontaneous coronary artery dissection: a case report and review of the literature. Cathet Cardiovasc Diagn 1998;45(3):280-286

32 Lee BW, Tay JS, Yip WC, Yap HK, Chan KY, Low PS. Kawasaki syndrome in Chinese children. Ann Trop Paediatr 1989;9(3):147-151

33 Taylor AJ, Byers JP, Cheitlin MD, Virmani R. Anomalous right or left coronary artery from the contralateral coronary sinus: "high-risk" abnormalities in the initial coronary artery course and heterogeneous clinical outcomes. Am Heart J 1997;133(4):428-435

34 Medic-Stojanoska M, Icin T, Pletikosic I, et al. Risk factors for accelerated atherosclerosis in young women with hyperprolactinemia. Med Hypotheses 2015;84(4):321-326

35 McCoy JM, Walkenhorst DE, McCauley KS, Elaasar H, Everett JR, Mix KS. Orphan nuclear receptor NR4A2 induces transcription of the immunomodulatory peptide hormone prolactin. J Inflamm (Lond) 2015;12:13

36 Barouch LA. Progress for peripartum cardiomyopathy. Trends Cardiovasc Med 2015;25(5):407-408

37 Hennighausen L, Robinson GW. Interpretation of cytokine signaling through the transcription factors STAT5A and STAT5B. Genes Dev 2008;22(6):711-721 